\title{
T-FUZZY MULTIPLY POSITIVE IMPLICATIVE BCC-IDEALS OF BCC-ALGEBRAS
}

\author{
JIANMING ZHAN and ZHISONG TAN
}

Received 22 October 2002

\begin{abstract}
The concept of fuzzy multiply positive BCC-ideals of BCC-algebras is introduced, and then some related results are obtained. Moreover, we introduce the concept of $T$-fuzzy multiply positive implicative BCC-ideals of BCC-algebras and investigate $T$-product of $T$-fuzzy multiply positive implicative BCC-ideals of BCC-algebras, examining its properties. Using a $t$-norm $T$, the direct product and $T$-product of $T$-fuzzy multiply positive implicative BCC-ideals of BCC-algebras are discussed and their properties are investigated.
\end{abstract}

2000 Mathematics Subject Classification: 06F35, 03G25, $03 B 52$.

1. Introduction and preliminaries. A BCK-algebra is an important class of logical algebras introduced by K. Iséki in 1966. After that, Iséki posed an interesting problem (solved by Wroński [8]) of whether the class of BCK-algebra is a variety. In connection with this problem, Komori [6] introduced a notion of BCC-algebras and Dudek [5] redefined it by using a dual form of the ordinary definition in the sense of Komori. In 1965, Zadeh introduced the notion of fuzzy sets [9]. At present, this concept has been applied to many mathematical branches such as group, functional analysis, probality theory and topology, and so on. In 1991, Ougen applied this concept to BCK-algebras [7], and also many fuzzy structures in BCC-algebras are considered. In this paper, the concept of fuzzy multiply positive implicative BCC-ideals of BCC-algebras is introduced, and some related results are obtained. Moreover, we introduce the concept of $T$-fuzzy multiply positive implicative BCC-ideals of BCC-algebras, investigating its properties. Using a $t$-norm $T$, the direct product and $T$-product of $T$-fuzzy multiply positive implicative BCC-ideals of BCC-algebras are discussed, and their properties are investigated.

By a BCC-algebra, we mean a nonempty set $G$ with a constant 0 and a binary operation $*$ satisfying the following conditions:

(I) $((x * y) *(z * y)) *(x * z)=0$,

(II) $x * x=0$,

(III) $0 * x=0$,

(IV) $x * 0=x$,

(V) $x * y=0$ and $y * x=0$ imply $x=y$ for all $x, y, z \in G$. 
On any BCC-algebra, one can define the partial ordering " $\leq$ " by putting $x \leq y$ if and only if $x * y=0$.

A BCK-algebra is a BCC-algebra, but there are not BCC-algebra which are not BCK-algebras (cf. [5]). Note that a BCC-algebra $X$ is a BCK-algebra if and only if it satisfies $(x * y) * z=(x * z) * y$ for all $x, y, z \in X$.

A nonempty subset $A$ of a BCC-algebra $G$ is called a BCC-ideal if (i) $0 \in A$ and (ii) $(x * y) * z \in A$ and $y \in A$ imply $x * z \in A$. For any elements $x$ and $y$ of a BCC-algebra, $x * y^{n}$ denotes $(\cdots((x * y) * y) * \cdots) * y$ in which $y$ occurs $n$ times. A nonempty subset $A$ of a BCC-algebra $G$ is called an $n$-fold BCC-ideal of $G$ if (i) $0 \in A$ and (ii) for every $x, y, z \in G$, there exists a natural number $n$ such that $x * z^{n} \in A$ whenever $(x * y) * z^{n} \in A$ and $y \in A$.

We now review some fuzzy logical concepts. A fuzzy set in set $G$ is a function $\mu: G \rightarrow[0,1]$. For a fuzzy set $\mu$ in $G$ and $\alpha \in[0,1]$, define $\mu_{\alpha}=\{x \in G \mid \mu(x) \geq$ $\alpha$ \} which is called a level set of $G$. A fuzzy set $\mu$ in a BCC-algebra $G$ is called a fuzzy BCC-ideal of $G$ if (i) $\mu(0) \geq \mu(x)$ and (ii) $\mu(x * z) \geq \min \{\mu((x * y) *$ $z), \mu(y)\}$ for all $x, y, z \in G$. A fuzzy set $\mu$ in a BCC-algebra $G$ is called an $n$ fold fuzzy BCC-ideal of $G$ if (i) $\mu(0) \geq \mu(x)$ for all $x \in G$ and (ii) for every $x, y, z \in G$, there exists a natural number $n$ such that $\mu\left(x * z^{n}\right) \geq \min \{\mu((x *$ $\left.\left.y) * z^{n}\right), \mu(y)\right\}$.

\section{Fuzzy multiply positive implicative BCC-ideals}

Definition 2.1. A nonempty subset $A$ of a BCC-algebra $G$ is called a multiply positive implicative BCC-ideal of $G$ if

(i) $0 \in A$,

(ii) for every $x, y, z \in X$, there exists a natural number $k=k(x, y, z)$ such that $x * z^{k} \in A$ whenever $(x * y) * z^{n} \in A$ and $y * z^{m} \in A$ for any natural numbers $m$ and $n$.

EXAMPLE 2.2. (i) Consider a BCC-algebra $G=\{0,1,2,3,4,5\}$ with the Cayley table as follows:

\begin{tabular}{l|llllll}
$*$ & 0 & 1 & 2 & 3 & 4 & 5 \\
\hline 0 & 0 & 0 & 0 & 0 & 0 & 0 \\
1 & 1 & 0 & 0 & 0 & 0 & 1 \\
2 & 2 & 2 & 0 & 0 & 1 & 1 \\
3 & 3 & 2 & 1 & 0 & 1 & 1 \\
4 & 4 & 4 & 4 & 4 & 0 & 1 \\
5 & 5 & 5 & 5 & 5 & 5 & 0
\end{tabular}

Then $G$ is a proper BCC-algebra since $(4 * 5) * 2 \neq(4 * 2) * 5$. It is routine to check that $A=\{0,1,2,3,4\}$ is a multiply positive implicative BCC-ideal of $G$. 
(ii) Consider a BCC-algebra $G=\{0, a, b, c, d\}$ with the Cayley table as follows:

\begin{tabular}{c|ccccc}
$*$ & 0 & $a$ & $b$ & $c$ & $d$ \\
\hline 0 & 0 & 0 & 0 & 0 & 0 \\
$a$ & $a$ & 0 & 0 & 0 & 0 \\
$b$ & $b$ & $b$ & 0 & 0 & 0 \\
$c$ & $c$ & $b$ & $a$ & 0 & $a$ \\
$d$ & $d$ & $d$ & $d$ & $d$ & 0
\end{tabular}

Then $G$ is a proper BCC-algebra since $(c * a) * d \neq(c * d) * a$. It is routine to check that $A=\{0, a, b, c\}$ is a multiply positive implicative BCC-ideal of $G$.

(iii) Consider a BCC-algebra $G=\{0, a, b, c, 1\}$ with the Cayley table as follows:

\begin{tabular}{c|ccccc}
$*$ & 0 & $a$ & $b$ & $c$ & 1 \\
\hline 0 & 0 & 0 & 0 & 0 & 0 \\
$a$ & $a$ & 0 & 0 & 0 & 0 \\
$b$ & $b$ & $b$ & 0 & 0 & 0 \\
$c$ & $c$ & $b$ & $a$ & 0 & $a$ \\
1 & 1 & $c$ & $c$ & $c$ & 0
\end{tabular}

Then $G$ is a proper BCC-algebra since $(1 * b) * a \neq(1 * a) * b$. Let $A=$ $\{0, b, c\}$, then $A$ is not a multiply positive implicative BCC-ideals of $G$ because $(1 * c) * 0^{n}=c * 0^{m}=c \in A$ while $1 * 0^{k}=1 \notin A$.

DEFINITION 2.3. A fuzzy set $\mu$ in a BCC-algebra $G$ is called a fuzzy multiply positive implicative BCC-ideal of $G$ if

(i) $\mu(0) \geq \mu(x)$ for all $x \in G$,

(ii) for any $n, m \in \mathbb{N}$, there exists a natural number $k=k(x, y, z)$ such that $\mu\left(x * z^{k}\right) \geq \min \left\{\mu\left((x * y) * z^{n}\right), \mu\left(y * z^{m}\right)\right\}$ for all $x, y, z \in G$.

EXAMPLE 2.4. (i) Consider a BCC-algebra $G=\{0,1,2,3,4\}$ with the Cayley table as follows:

\begin{tabular}{l|lllll}
$*$ & 0 & 1 & 2 & 3 & 4 \\
\hline 0 & 0 & 0 & 0 & 0 & 0 \\
1 & 1 & 0 & 1 & 0 & 0 \\
2 & 2 & 2 & 0 & 0 & 0 \\
3 & 3 & 3 & 1 & 0 & 0 \\
4 & 4 & 3 & 4 & 3 & 0
\end{tabular}

It is a proper BCC-algebra since $(3 * 1) * 2 \neq(3 * 2) * 1$. Define a fuzzy set $\mu$ in $G$ by $\mu(4)=0.3$ and $\mu(x)=0.8$ for all $x \neq 4$. Then $\mu$ is a fuzzy multiply positive implicative BCC-ideal of $G$. 
(ii) Let $G$ be a proper BCC-algebra as (i) and let $\mu$ be a fuzzy set in $G$ defined by

$$
\mu(x)= \begin{cases}\alpha_{1} & \text { if } x \in\{0,2,3\} \\ \alpha_{2} & \text { otherwise }\end{cases}
$$

where $\alpha_{1}>\alpha_{2}$ in $[0,1]$. It is easy to check that $\mu$ is not a fuzzy multiply positive implicative BCC-ideal of $G$ because $\mu\left(4 * 0^{k}\right)=\mu(4)=\alpha_{2} \leq \min \{\mu((4 * 3) *$ $\left.\left.0^{n}\right), \mu\left(3 * 0^{m}\right)\right\}$ for any positive integer numbers $m, n$, and $k$.

THEOREM 2.5. Let $\mu$ be a fuzzy set in a BCC-algebra $G$, then $\mu$ is a fuzzy multiply positive implicative BCC-ideal of $G$ if and only if the nonempty level set $\mu_{\alpha}=\{x \in G \mid \mu(x) \geq \alpha\}$ of $\mu$ is a multiply positive implicative BCC-ideal of $G$.

Proof. Suppose that $\mu$ is a fuzzy multiply positive implicative BCC-ideal of $G$ and $\mu_{\alpha} \neq \varnothing$ for any $\alpha \in[0,1]$. Then there exists $x \in \mu_{\alpha}$ and so $\mu(x) \geq \alpha$. It follows that $\mu(0) \geq \mu(x) \geq \alpha$ so that $0 \in \mu_{\alpha}$. Let $x, y, z \in G$ be such that $(x * y) * z^{n} \in \mu_{\alpha}$ and $y * z^{m} \in \mu_{\alpha}$. By Definition 2.3, there exists a natural number $k$ such that $\mu\left(x * z^{k}\right) \geq \min \left\{\mu\left((x * y) * z^{n}\right), \mu\left(y * z^{m}\right)\right\} \geq \min \{\alpha, \alpha\}=$ $\alpha$ and that $x * z^{k} \in \mu_{\alpha}$. Hence $\mu_{\alpha}$ is a multiply positive implicative BCC-ideal of $G$. Conversely, assume that $\mu_{\alpha}$ is a multiply positive implicative BCC-ideal of $G$ for every $\alpha \in[0,1]$. For any $x \in G$, let $\mu(x)=\alpha$. Then $x \in \mu_{\alpha}$. Since $0 \in \mu_{\alpha}$, it follows that $\mu(0) \geq \alpha=\mu(x)$ so that $\mu(0) \geq \mu(x)$ for all $x \in G$. Now suppose that there exist $x_{0}, y_{0}, z_{0} \in G$ such that $\mu\left(x_{0} * z_{0}^{k}\right)<\min \left\{\mu\left(\left(x_{0} * y_{0}\right) *\right.\right.$ $\left.\left.z_{0}\right), \mu\left(y_{0} * z_{0}^{m}\right)\right\}$. Let $\lambda_{0}=\left(\mu\left(x_{0} * z_{0}^{k}\right)+\min \left\{\mu\left(\left(x_{0} * y_{0}\right) * z_{0}\right), \mu\left(y_{0} * z_{0}^{m}\right)\right\}\right) / 2$, then $\lambda_{0}>\mu\left(x_{0} * z_{0}^{k}\right)$ and $0 \leq \lambda_{0}<\min \left\{\mu\left(\left(x_{0} * y_{0}\right) * z_{k}^{n}\right), \mu\left(y_{0} * z_{0}^{m}\right)\right\} \leq 1$, so we have $\mu\left(\left(x_{0} * y_{0}\right) * z_{0}^{n}\right) \geq \lambda_{0}$ and $\mu\left(y_{0} * z_{0}^{m}\right) \geq \lambda_{0}$, then $\left(x_{0} * y_{0}\right) * z_{0}^{n} \in \mu_{\lambda_{0}}$ and $y_{0} * z_{0}^{n} \in \mu_{\lambda_{0}}$. As $\mu_{\lambda_{0}}$ is a multiply positive BCC-ideal of $G$, it implies $x_{0} * z_{0}^{k} \in \mu_{\lambda_{0}}$ and $\mu\left(x_{0} * z_{0}^{k}\right) \geq \lambda_{0}$. This is a contradiction. Hence $\mu$ is a fuzzy multiply positive implicative BCC-ideal of $G$.

THEOREM 2.6. Let $A$ be a nonempty subset of a BCC-algebra $G$, and $\mu$ a fuzzy set in $G$ defined by

$$
\mu(x)= \begin{cases}\alpha_{1} & \text { if } x \in A, \\ \alpha_{2} & \text { otherwise, }\end{cases}
$$

where $\alpha_{1}>\alpha_{2}$ in $[0,1]$. Then $\mu$ is a fuzzy multiply positive implicative BCC-ideal of $G$ if and only if $A$ is a multiply positive implicative BCC-ideal of $G$.

Proof. Assume that $\mu$ is a fuzzy multiply positive implicative BCC-ideal of $G$. Since $\mu(0) \geq \mu(x)$ for all $x \in G$, we have $\mu(0)=\alpha_{1}$ and so $0 \in A$. Let $x, y, z \in G$ be such that $(x * y) * z^{n} \in A$ and $y * z^{m} \in A$. By Definition 2.3, there exists a natural number $k=k(x, y, z)$ such that $\mu\left(x * z^{k}\right) \geq \min \{\mu((x *$ $\left.\left.y) * z^{n}\right), \mu\left(y * z^{m}\right)\right\}=\alpha_{1}$ and that $x * z^{k} \in A$. Hence $A$ is a multiply positive implicative BCC-ideal of $G$. 
Conversely, suppose that $A$ is a multiply positive implicative BCC-ideal of $G$. Since $0 \in A$, it follows that $\mu(0)=\alpha_{1} \geq \mu(x)$ for all $x \in G$. Let $x, y, z \in$ $G$. If $y * z^{m} \notin A$ and $(x * y) * z^{n} \in A$, then clearly $\mu\left(x * z^{k}\right) \geq \min \{\mu((x *$ $\left.\left.y) * z^{n}\right), \mu\left(y * z^{m}\right)\right\}$. Assume that $y * z^{m} \in A$ and $(x * y) * z^{n} \notin A$, we have $(x * y) * z^{k} \notin A$. Therefore $\mu\left(x * z^{k}\right)=\alpha_{2}=\min \left\{\mu\left((x * y) * z^{n}\right), \mu\left(y * z^{m}\right)\right\}$. Hence, $\mu$ is a fuzzy multiply positive implicative BCC-ideal of $G$.

A fuzzy relation on any set $S$ is a fuzzy subset $\mu: S \times S \rightarrow[0,1]$. If $\mu$ is a fuzzy relation on a set $S$ and $v$ is a fuzzy subset of $S$, then $\mu$ is a fuzzy relation on $v$ if $\mu(x, y) \leq \min \{v(x), v(y)\}$ for all $x, y \in S$. Let $\mu$ and $v$ on $S$ be defined as $(\mu \times v)(x, y)=\min \{\mu(x), v(y)\}$. One can prove that $\mu \times v$ is a fuzzy relation on $S$ and $(\mu \times v)_{t}=\mu_{t} \times v_{t}$ for all $t \in[0,1]$. If $\mu$ is a fuzzy subset of a set $S$, the strongest fuzzy relation on $S$ that is a fuzzy relation on $v$ is $\mu_{v}$, given by $\mu_{v}(x, y)=\min \{\mu(x), v(y)\}$ for all $x, y \in S$. In this case we have $\left(\mu_{v}\right)_{t}=v_{t} \times v_{t}$ for all $t \in[0,1]$ (see [2]).

THEOREM 2.7. For a given fuzzy subset $v$ of a BCC-algebra $G$, let $\mu_{v}$ be the strongest fuzzy relation on $G$. If $\mu_{v}$ is a fuzzy multiply positive implicative BCCideal of $G \times G$, then $v(0) \geq v(x)$ for all $x \in G$.

Proof. Since $\mu_{v}$ is a fuzzy multiply positive implicative BCC-ideal of $G \times G$, it follows that $\mu_{v}(0,0) \geq \mu_{v}(x, x)$ for all $x \in G$. This means that $\min \{v(0)$, $v(0)\} \geq \min \{v(x), v(x)\}$, which implies that $v(0) \geq v(x)$.

THEOREM 2.8. If $v$ is a fuzzy multiply positive implicative BCC-ideal of a $B C C$-algebra $G$, then the level multiply positive implicative BCC-ideals of $\left(\mu_{v}\right)_{t}$ are given by

$$
\left(\mu_{v}\right)_{t}=\mu_{t} \times v_{t} \quad \forall t \in[0,1]
$$

The proof is obvious.

THEOREM 2.9. If $\mu$ and $v$ are fuzzy multiply positive implicative BCC-ideals of a BCC-algebra $G$, then $\mu \times v$ is a fuzzy multiply positive implicative BCC-ideal of $G \times G$.

Proof. For any $(x, y) \in G \times G$,

$$
\begin{aligned}
(\mu \times v)(0,0) & =\min \{\mu(0), v(0)\} \geq \min \{\mu(x), \nu(x)\} \\
& =(\mu \times v)(x, y) .
\end{aligned}
$$

Now, let $x=\left(x_{1}, x_{2}\right), y=\left(y_{1}, y_{2}\right)$, and $z=\left(z_{1}, z_{2}\right) \in G \times G$. For any $n, m \in \mathbb{N}$, there exists a natural number $k$ such that

$$
\begin{aligned}
(\mu \times v)\left(x * z^{k}\right) & =(\mu \times v)\left(\left(x_{1}, x_{2}\right) *\left(z_{1}, z_{2}\right)^{k}\right) \\
& =(\mu \times v)\left(x_{1} * z_{1}^{k}, x_{2} * z_{2}^{k}\right) \\
& =\min \left\{\mu\left(x_{1} * z_{1}^{k}\right), v\left(x_{2} * z_{2}^{k}\right)\right\}
\end{aligned}
$$




$$
\begin{gathered}
\geq \min \left\{\min \left\{\mu\left(\left(x_{1} * y_{1}\right) * z_{1}^{n}\right), \mu\left(y_{1} * z_{1}^{m}\right)\right\},\right. \\
\left.\min \left\{v\left(\left(x_{1} * y_{2}\right) * z_{2}^{n}\right), v\left(y_{2} * z_{2}^{m}\right)\right\}\right\} \\
=\min \left\{\min \left\{\mu\left(\left(x_{1} * y_{1}\right) * z_{1}^{n}\right), v\left(\left(x_{2} * y_{2}\right) * z_{2}^{n}\right)\right\},\right. \\
\left.\min \left\{\mu\left(y_{1} * z_{1}^{m}\right), v\left(y_{2} * z_{2}^{m}\right)\right\}\right\} \\
=\min \left\{(\mu \times v)\left(\left(\left(x_{1}, x_{2}\right) *\left(y_{1}, y_{2}\right)\right) *\left(z_{1}, z_{2}\right)^{n}\right),\right. \\
\left.(\mu \times v)\left(\left(y_{1}, y_{2}\right) *\left(z_{1}, z_{2}\right)^{m}\right)\right\} \\
=\min \left\{(\mu \times v)\left((x * y) * z^{n}\right),(\mu \times v)\left(y * z^{m}\right)\right\} .
\end{gathered}
$$

Hence $\mu \times v$ is a fuzzy multiply positive implicative BCC-ideals of $G \times G$.

THEOREM 2.10. Let $\mu$ and $v$ be fuzzy subsets of a BCC-algebra $G$ such that $\mu \times v$ is a fuzzy multiply positive implicative BCC-ideal of $G \times G$. Then

(i) either $\mu(x) \leq \mu(0)$ or $v(x) \leq v(0)$ for all $x \in G$,

(ii) if $\mu(x) \leq \mu(0)$ for all $x \in G$, then either $\mu(x) \leq \nu(0)$ or $v(x) \leq v(0)$,

(iii) if $v(x) \leq v(0)$ for all $x \in G$, then either $\mu(x) \leq \mu(0)$ or $v(x) \leq \mu(0)$,

(iv) either $\mu$ or $v$ is a fuzzy multiply positive implicative BCC-ideal of $G$.

Proof. (i) Suppose that $\mu(x)>\mu(0)$ and $\nu(x)>v(0)$ for some $x, y \in G$. Then $(\mu \times v)(x, y)=\min \{\mu(x), v(y)\}>\min \{\mu(0), \nu(0)\}=(\mu \times v)(0,0)$. This is a contradiction and we obtain (i).

(ii) Assume that there exist $x, y \in G$ such that $\mu(x)>v(0)$ and $v(y)>v(0)$. Then $(\mu \times v)(0,0)=\min \{\mu(0), v(0)\}=v(0)$. It follows that $(\mu \times v)(x, y)=$ $\min \{\mu(x), v(y)\}>v(0)=(\mu \times v)(0,0)$. This is a contradiction. Hence (ii) holds.

(iii) Item (iii) is proved by similar method to part (ii).

(iv) Since by (i), either $\mu(x) \leq \mu(0)$ or $v(x) \leq \nu(0)$ for all $x \in G$, without loss of generality, we may assume that $v(x) \leq v(0)$ for all $x \in G$. Form (iii), it follows that either $\mu(x) \leq \mu(0)$ or $v(x) \leq \mu(0)$. If $v(x) \leq \mu(0)$ for all $x \in G$, then $(\mu \times v)(0, x)=\min \{\mu(0), v(x)\}=v(x)$. Let $\left(x_{1}, x_{2}\right),\left(y_{1}, y_{2}\right),\left(z_{1}, z_{2}\right) \in$ $G \times G$. Since $\mu \times v$ is a fuzzy multiply positive implicative BCC-ideal of $G \times G$, then for any $n, m \in \mathbb{N}$, there exists a natural number $k$ such that

$$
\begin{gathered}
(\mu \times v)\left(\left(x_{1}, x_{2}\right) *\left(z_{1}, z_{2}\right)^{k}\right) \\
\geq \min \left\{(\mu \times v)\left(\left(\left(x_{1}, x_{2}\right) *\left(y_{1}, y_{2}\right)\right) *\left(z_{1}, z_{2}\right)^{n}\right),\right. \\
\left.(\mu \times v)\left(\left(y_{1}, y_{2}\right) *\left(z_{1}, z_{2}\right)^{m}\right)\right\} \\
=\min \left\{(\mu \times v)\left(\left(\left(x_{1} * y_{1}\right) * z_{1}^{n}\right),\left(\left(x_{2} * y_{2}\right) * z_{2}^{n}\right)\right),\right. \\
\left.(\mu \times v)\left(y_{1} * z_{1}^{m}, y_{2} * z_{2}^{m}\right)\right\} .
\end{gathered}
$$


If we take $x_{1}=y_{1}=z_{1}=0$, then

$$
\begin{aligned}
v\left(x_{2} * z_{2}^{k}\right) & =(\mu \times v)\left(0, x_{2} * z_{2}^{k}\right) \\
& =(\mu \times v)\left(\left(0, x_{2}\right) *\left(0, z_{2}\right)^{k}\right) \\
& \geq \min \left\{(\mu \times v)\left(0,\left(x_{2} * y_{2}\right) * z_{2}^{n}\right),(\mu \times v)\left(0, y_{2} * z_{2}^{m}\right)\right\} \\
& =\min \left\{\min \left\{\mu(0), v\left(\left(x_{2} * y_{2}\right) * z_{2}^{n}\right)\right\}, \min \left\{v(0), v\left(y_{2} * z_{2}^{m}\right)\right\}\right\} \\
& =\min \left\{v\left(\left(x_{2} * y_{2}\right) * z_{2}^{n}\right), v\left(y_{2} * z_{2}^{m}\right)\right\} .
\end{aligned}
$$

This proves that $v$ is a fuzzy multiply positive BCC-ideal of $G$. Now we consider the case $\mu(x) \leq \mu(0)$ for all $x \in G$. Suppose that $\nu(y)>\mu(0)$ for some $y \in G$. Then $v(0) \geq v(y)>\mu(0)$. Since $\mu(0) \geq \mu(x)$ for all $x \in G$, it follows that $\nu(0)>\mu(x)$ for any $x \in G$. Hence $(\mu \times v)(x, 0)=\min \{\mu(x), v(0)\}=\mu(x)$. Taking $x_{2}=y_{2}=z_{2}=0$ in (2.6), then

$$
\begin{aligned}
\mu\left(x_{1} * z_{1}^{k}\right) & =(\mu \times v)\left(x_{1} * z_{1}^{k}, 0\right) \\
& =(\mu \times v)\left(\left(x_{1}, 0\right) *\left(z_{1}, 0\right)^{k}\right) \\
& \geq \min \left\{(\mu \times v)\left(\left(x_{1} * y_{1}\right) * z_{1}^{n}, 0\right),(\mu \times v)\left(y_{1} * z_{1}^{m}, 0\right)\right\} \\
& =\min \left\{\min \left\{\mu\left(\left(x_{1} * y_{1}\right) * z_{1}^{n}\right), v(0)\right\}, \min \left\{\mu\left(y_{1} * z_{1}^{m}\right), v(0)\right\}\right\} \\
& =\min \left\{\mu\left(\left(x_{1} * y_{1}\right) * z_{1}^{n}\right), \mu\left(y_{1} * z_{1}^{m}\right)\right\}
\end{aligned}
$$

which proves that $\mu$ is a fuzzy multiply positive implicative BCC-ideal of $G$.

THEOREM 2.11. Let $v$ be a fuzzy subset of a BCC-algebra $G$ and let $\mu_{v}$ be the strongest fuzzy relation on $G$. Then $v$ is a fuzzy multiply positive implicative $B C C$-ideal of $G$ if and only if $\mu_{v}$ is a fuzzy multiply positive implicative BCC-ideal of $G \times G$.

Proof. Assume that $v$ is a fuzzy multiply positive implicative BCC-ideal of $X$, then

$$
\mu_{v}(0,0)=\min \{v(0), v(0)\} \geq \min \{v(x), v(y)\}=\mu_{v}(x, y)
$$

for any $(x, y) \in G \times G$. Moreover, for any $n, m \in \mathbb{N}$, there exists a natural number $k$ such that

$$
\begin{aligned}
\mu_{v}\left(\left(x_{1}, x_{2}\right) *\left(z_{1}, z_{2}\right)^{k}\right)= & \mu_{v}\left(x_{1} * z_{1}^{k}, x_{2} * z_{2}^{k}\right) \\
= & \min \left\{v\left(x_{1} * z_{1}^{k}\right), v\left(x_{2} * z_{2}^{k}\right)\right\} \\
\geq & \min \left\{\min \left\{v\left(\left(x_{1} * y_{1}\right) * z_{1}^{n}\right), v\left(y_{1} * z_{1}^{m}\right)\right\},\right. \\
& \left.\quad \min \left\{v\left(\left(x_{2} * y_{2}\right) * z_{2}^{n}\right), v\left(y_{2} * z_{2}^{m}\right)\right\}\right\}
\end{aligned}
$$




$$
\begin{gathered}
=\min \left\{\min \left\{v\left(\left(x_{1} * y_{1}\right) * z_{1}^{n}\right), v\left(\left(x_{2} * y_{2}\right) * z_{2}^{n}\right)\right\},\right. \\
\left.\min \left\{v\left(y_{1} * z_{1}^{m}\right), v\left(y_{2} * z_{2}^{m}\right)\right\}\right\} \\
=\min \left\{\mu_{v}\left(\left(\left(x_{1} * y_{1}\right) * z_{1}^{n}\right),\left(x_{2} * y_{2}\right) * z_{2}^{n}\right),\right. \\
\left.\mu_{v}\left(y_{1} * z_{1}^{m}, y_{2} * z_{2}^{m}\right)\right\} \\
=\min \left\{\mu_{v}\left(\left(\left(x_{1}, x_{2}\right) *\left(y_{1}, y_{2}\right)\right) *\left(z_{1}, z_{2}\right)^{n}\right),\right. \\
\left.\mu_{v}\left(\left(y_{1}, y_{2}\right) *\left(z_{1}, z_{2}\right)^{m}\right)\right\}
\end{gathered}
$$

for any $\left(x_{1}, x_{2}\right),\left(y_{1}, y_{2}\right),\left(z_{1}, z_{2}\right) \in G \times G$.

Hence $\mu_{v}$ is a fuzzy multiply positive implicative BCC-ideal of $G \times G$.

Conversely, suppose that $\mu_{v}$ is a fuzzy multiply positive implicative BCCideal of $G \times G$. Then for all $\left(x_{1}, x_{2}\right) \in G \times G$,

$$
\min \{v(0), v(0)\}=\mu_{v}(0,0) \geq \mu_{v}\left(x_{1}, x_{2}\right)=\min \left\{v\left(x_{1}\right), v\left(x_{2}\right)\right\}
$$

It follows that $v(0) \geq v(x)$ for all $x \in G$. Now, for any $n, m \in \mathbb{N}$, there exists a natural number $k$ such that

$$
\begin{aligned}
\min & \left\{v\left(x_{1} * z_{1}^{k}\right), v\left(x_{2} * z_{2}^{k}\right)\right\} \\
= & \mu_{v}\left(x_{1} * z_{1}^{k}, x_{2} * z_{2}^{k}\right)=\mu_{v}\left(\left(x_{1}, x_{2}\right) *\left(z_{1}, z_{2}\right)^{k}\right) \\
\geq & \min \left\{\mu_{v}\left(\left(\left(x_{1}, x_{2}\right) *\left(y_{1}, y_{2}\right)\right) *\left(z_{1}, z_{2}\right)^{n}\right), \mu_{v}\left(\left(y_{1}, y_{2}\right) *\left(z_{1}, z_{2}\right)^{m}\right)\right\} \\
= & \min \left\{\mu_{v}\left(\left(x_{1} * y_{1}\right) * z_{1}^{n},\left(x_{2} * y_{2}\right) * z_{2}^{n}\right), \mu_{v}\left(y_{1} * z_{1}^{m}, y_{2} * z_{2}^{m}\right)\right\} \\
= & \min \left\{\min \left\{v\left(\left(x_{1} * y_{1}\right) * z_{1}^{n}\right), v\left(\left(x_{2} * y_{2}\right) * z_{2}^{n}\right)\right\},\right. \\
= & \left.\min \left\{v\left(y_{1} * z_{1}^{m}\right), v\left(y_{2} * z_{2}^{m}\right)\right\}\right\} \\
& \min \left\{v\left(\left(x_{1} * y_{1}\right) * z_{1}^{n}\right), v\left(y_{1} * z_{1}^{m}\right)\right\}, \\
& \left.\min \left\{v\left(\left(x_{2} * y_{2}\right) * z_{2}^{n}\right), v\left(y_{2} * z_{2}^{m}\right)\right\}\right\} .
\end{aligned}
$$

If we take $x_{2}=y_{2}=z_{2}=0$ (resp., $x_{1}=y_{1}=z_{1}=0$ ), then $v\left(x_{1} * z_{1}^{k}\right) \geq$ $\min \left\{v\left(\left(x_{1} * y_{1}\right) * z_{1}^{n}\right), v\left(y_{2} * z_{2}^{m}\right)\right\}$. Hence $v$ is a fuzzy multiply positive implicative BCC-ideal of $G$.

\section{3. $T$-fuzzy multiply positive implicative BCC-ideals}

Definition $3.1[1]$. By a $t$-norm $T$, we mean a function $T:[0,1] \times[0,1] \rightarrow$ $[0,1]$ satisfying the following conditions:

(I) $T(x, 1)=x$,

(II) $T(x, y) \leq T(x, z)$ if $y \leq z$,

(III) $T(x, y)=T(y, x)$,

(IV) $T(x, T(y, z))=T(T(x, y), z)$ for all $x, y, z \in[0,1]$.

Every $t$-norm $T$ has a useful property $T(\alpha, \beta) \leq \min \{\alpha, \beta\}$ for all $\alpha, \beta \in[0,1]$. 
LEMMA 3.2 [1]. Let $T$ be a $t$-norm. Then $T(T(\alpha, \beta), T(\nu, \delta))=T(T(\alpha, v)$, $T(\beta, \delta))$ for all $\alpha, \beta, \nu, \delta \in[0,1]$.

Definition 3.3. A fuzzy subset $\mu: G \rightarrow[0,1]$ in a BCC-algebra $G$ is called a fuzzy multiply positive implicative BCC-ideal of $G$ with respect to a $t$-norm $T$ (briefly, $T$-fuzzy multiply positive implicative BCC-ideal of $G$ ) if

(i) $\mu(0) \geq \mu(x)$ for all $x \in G$,

(ii) for any $n, m \in \mathbb{N}$, there exists a natural number $k=k(x, y, z)$ such that $\mu\left(x * z^{k}\right) \geq T\left(\mu\left((x * y) * z^{n}\right), \mu\left(y * z^{m}\right)\right)$ for any $x, y, z \in G$.

EXAMPLE 3.4. Consider a BCC-algebra $G=\{0,1,2,3,4\}$ with the Cayley table as follows:

\begin{tabular}{l|lllll}
$*$ & 0 & 1 & 2 & 3 & 4 \\
\hline 0 & 0 & 0 & 0 & 0 & 0 \\
1 & 1 & 0 & 1 & 0 & 0 \\
2 & 2 & 2 & 0 & 0 & 0 \\
3 & 3 & 3 & 1 & 0 & 0 \\
4 & 3 & 4 & 4 & 3 & 0
\end{tabular}

By routine calculation, $G$ is a proper BCC-algebra (cf. [5]). Define a fuzzy set $\mu$ by $\mu(0)=\mu(1)=\mu(2)=\mu(3)=0.8$ and $\mu(4)=0.3$. Let $T(\alpha, \beta)=\max \{\alpha+$ $\beta-1,0\}$ for all $\alpha, \beta \in[0,1]$. Then $T$ is a $t$-norm. It is easy to check that $\mu$ is a $T$-fuzzy multiply positive implicative BCC-ideal of $G$.

THEOREM 3.5. Let $\mu$ be a T-fuzzy multiply positive implicative BCC-ideal of a BCC-algebra $G$ and let $\alpha \in[0,1]$ if $\alpha=1$, then the nonempty subset $\mu_{\alpha}$ is a multiply positive implicative $B C C$-ideal of $G$.

Proof. Assume that $\alpha=1$ and $x \in \mu_{\alpha}$, then $\mu(x) \geq 1$. Thus $\mu(0) \geq \mu(x) \geq 1$ and $0 \in \mu_{\alpha}$.

Moreover, suppose that $(x * y) * z^{n} \in \mu_{\alpha}$ and $y * z^{m} \in \mu_{\alpha}$, then $\mu((x * y) *$ $\left.z^{n}\right) \geq 1$ and $\mu\left(y * z^{m}\right) \geq 1$. By Definition 3.3, there exists a natural number $k$ such that $\mu\left(x * z^{k}\right) \geq T\left(\mu\left((x * y) * z^{n}\right), \mu\left(y * z^{m}\right)\right) \geq T(1,1)=1$ and that $x * z^{k} \in \mu_{\alpha}$. Hence $\mu_{\alpha}$ is a multiply positive implicative BCC-ideal of $G$.

For a fuzzy set $\mu$ on a BCC-algebra $G$ and a map $\theta: G \rightarrow G$, we define a mapping $\mu[\theta]: G \rightarrow[0,1]$ by $\mu[\theta](x)=\mu(\theta(x))$ for all $x \in G$.

THEOREM 3.6. If $\mu$ is a T-fuzzy multiply positive implicative BCC-ideal of a $B C C$-algebra $G$ and $\theta$ is an epimorphism of $G$, then $\mu[\theta]$ is a $T$-fuzzy multiply positive implicative BCC-ideal of $G$. 
Proof. Let $\mu[\theta](0)=\mu(\theta(0))=\mu(0) \geq \mu(y)$ for any $y \in G$. Since $\theta$ is an epimorphism of $G$, then there exists $x \in G$ such that $\theta(x)=y$. Thus $\mu[\theta](0) \geq$ $\mu(\theta(x))=\mu[\theta](x)$. As $y$ is an arbitrary element of $G$, the above result is true for any $x \in G$.

Moreover, for any $n, m \in \mathbb{N}$, there exists a natural number $k$ such that

$$
\begin{aligned}
\mu[\theta]\left(x * z^{k}\right) & =\mu\left(\theta\left(x * z^{k}\right)\right)=\mu\left(\theta(x) * \theta(z)^{k}\right) \\
& \geq T\left(\mu\left((\theta(x) * \theta(y)) * \theta(z)^{n}\right), \mu\left(\theta(y) * \theta(z)^{m}\right)\right) \\
& =T\left(\mu\left(\theta\left((x * y) * z^{n}\right)\right), \mu\left(\theta\left(y * z^{m}\right)\right)\right) \\
& =T\left(\mu[\theta]\left((x * y) * z^{n}\right), \mu[\theta]\left(y * z^{m}\right)\right) .
\end{aligned}
$$

Hence $\mu[\theta]$ is a $T$-fuzzy multiply positive implicative BCC-ideal of $G$.

Let $f$ be a mapping defined on a BCC-algebra $G$. If $v$ is a fuzzy set in $f(G)$, then the fuzzy set $\mu_{v}$ of $G$ defined by $\mu(x)=v(f(x))$ is called the preimage of $v$ under $f$.

THEOREM 3.7. An onto homomorphic preimage of a T-fuzzy multiply positive implicative BCC-ideal is a T-fuzzy multiply positive implicative BCC-ideal.

Proof. Let $f: G \rightarrow G^{\prime}$ be an onto homomorphism of BCC-algebra, $v$ a $T$ fuzzy multiply positive implicative BCC-ideal of $G^{\prime}$, and $\mu$ the preimage of $v$ under $f$. Then $\mu(0)=v(f(0))=v\left(0^{\prime}\right) \geq \nu(f(x))=\mu(x)$ for all $x \in G$. Moreover, for any $n, m \in \mathbb{N}$, there exists a natural number $k$ such that

$$
\begin{aligned}
\mu\left(x * z^{k}\right) & =v\left(f\left(x * z^{k}\right)\right)=v\left(f(x) * f(z)^{k}\right) \\
& \geq T\left(v\left((f(x) * f(y)) * f(z)^{n}\right), v\left(f(y) * f(z)^{m}\right)\right) \\
& =T\left(\nu\left(f\left((x * y) * z^{n}\right)\right), v\left(f\left(y * z^{m}\right)\right)\right) \\
& =T\left(\mu\left((x * y) * z^{n}\right), \mu\left(y * z^{m}\right)\right)
\end{aligned}
$$

for any $x, y, z \in G$. Hence $\mu$ is a $T$-fuzzy multiply positive implicative BCC-ideal of $G$.

If $\mu$ is a fuzzy set in a BCC-algebra $G$ and $f$ is a mapping defined on $G$, then the fuzzy set $\mu^{f}$ in $f(G)$ defined by $\mu^{f}(y)=\sup _{x \in f^{-1}(y)} \mu(x)$ for all $y \in G$ is called the image of $\mu$ under $f$. A fuzzy set $\mu$ in $G$ is said to have sup property if, for every subset $T \subseteq G$, there exists $t_{0} \in T$ such that $\mu\left(t_{0}\right)=\sup _{t \in T} \mu(t)$.

THEOREM 3.8. An onto homomorphic image of a T-fuzzy multiply positive implicative BCC-ideal with sup property is a T-fuzzy multiply positive implicative BCC-ideal.

Proof. Let $f: G \rightarrow G^{\prime}$ be an onto homomorphism of BCC-algebras and let $\mu$ be a $T$-fuzzy multiply positive implicative BCC-ideal of $G$ with sup property. Then $\mu^{f}(0)=\sup _{f \in f^{-1}(0)} \mu(t)=\mu(0) \geq \mu(x)$ for any $x \in G$. Furthermore, we 
have $\mu^{f}\left(x_{1}\right)=\sup _{t \in f^{-1}\left(x_{1}\right)} \mu(t)$ for any $x_{1} \in G^{\prime}$. Thus $\mu^{f}(0) \geq \sup _{t \in f^{-1}\left(x_{1}\right)} \mu(t)=$ $\mu^{f}\left(x_{1}\right)$ for any $x_{1} \in G^{\prime}$. Moreover, for any $x_{1}, y_{1}, z_{1} \in G^{\prime}$, let $x \in f^{-1}\left(x_{1}\right)$, $y \in f^{-1}\left(y_{1}\right)$, and $z \in f^{-1}\left(z_{1}\right)$ such that

$$
\begin{aligned}
\mu\left(x * z^{k}\right) & =\sup _{t \in f^{-1}\left(x_{1} * z_{1}^{k}\right)} \mu(t), \\
\mu\left((x * y) * z^{n}\right) & =\sup _{t \in f^{-1}\left((x * y) * z^{n}\right)} \mu(t), \\
\mu\left(y * z^{n}\right) & =\sup _{t \in f^{-1}\left(y_{1} * z_{1}^{m}\right)} \mu(t) .
\end{aligned}
$$

Thus

$$
\begin{aligned}
\mu^{f}\left(x_{1} * z_{1}^{k}\right) & =\sup _{t \in f^{-1}\left(x_{1} * z_{1}^{k}\right)} \mu(t)=\mu\left(x * z^{k}\right) \\
& \geq T\left(\mu\left((x * y) * z^{n}\right), \mu\left(y * z^{m}\right)\right) \\
& =T\left(\sup _{t \in f^{-1}\left(\left(x_{1} * y_{1}\right) * z_{1}^{n}\right)} \mu(t), \sup _{t \in f^{-1}\left(y_{1} * z_{1}^{m}\right)} \mu(t)\right) \\
& =T\left(\mu^{f}\left(\left(x_{1} * y_{1}\right) * z_{1}^{n}\right), \mu^{f}\left(y_{1} * z_{1}^{m}\right)\right) .
\end{aligned}
$$

Therefore, $\mu^{f}$ is a $T$-fuzzy multiply positive implicative BCC-ideal of $G^{\prime}$.

\section{Fuzzy multiply positive implicative BCC-ideals induced by norms}

THEOREM 4.1. Let $T$ be a $t$-norm and $G=G_{1} \times G_{2}$ the direct product BCCalgebra of BCC-algebras $G_{1}$ and $G_{2}$. If $\mu_{1}$ (resp., $\mu_{2}$ ) is a $T$-fuzzy multiply positive implicative BCC-ideal of $G_{1}$ (resp., $G_{2}$ ), then $\mu=\mu_{1} \times \mu_{2}$ is a $T$-fuzzy multiply positive implicative BCC-ideal of $G$ defined by $\mu\left(x_{1}, x_{2}\right)=\left(\mu_{1} \times \mu_{2}\right)\left(x_{1}, x_{2}\right)=$ $T\left(\mu_{1}\left(x_{1}\right), \mu_{2}\left(x_{2}\right)\right)$ for all $\left(x_{1}, x_{2}\right) \in G_{1} \times G_{2}$.

The proof is identical with the corresponding proof from [3].

We will generalize the idea to the product of $n T$-fuzzy multiply positive implicative BCC-ideals. We first need to generalize the domain of $t$-norm $T$ to $\Pi_{i=1}^{n}[0,1]$ as follows.

The function $T_{n}: \Pi_{i=1}^{n}[0,1] \rightarrow[0,1]$ is defined by

$$
T_{n}\left(\alpha_{1}, \alpha_{2}, \ldots, \alpha_{n}\right)=T\left(\alpha_{i}, T_{n-1}\left(\alpha_{1}, \ldots, \alpha_{i-1}, \alpha_{i+1}, \ldots, \alpha_{n}\right)\right)
$$

for all $1 \leq i \leq n$, where $n \geq 2, T_{2}=T$, and $T_{1}=$ id (identity). For a $t$-norm $T$ and every $\alpha_{i}, \beta_{i} \in[0,1]$, where $1 \leq i \leq n$ and $n \geq 2$, we have

$$
\begin{aligned}
& T_{n}\left(T\left(\alpha_{1}, \beta_{1}\right), T\left(\alpha_{2}, \beta_{2}\right), \ldots, T\left(\alpha_{n}, \beta_{n}\right)\right) \\
& \quad=T\left(T_{n}\left(\alpha_{1}, \alpha_{2}, \ldots, \alpha_{n}\right), T_{n}\left(\beta_{1}, \beta_{2}, \ldots, \beta_{n}\right)\right) .
\end{aligned}
$$


THEOREM 4.2. Let $T$ be a $t$-norm, $\left\{G_{i}\right\}_{i=1}^{n}$ the finite collection of BCC-algebras, and $G=\Pi_{n}^{i=1} G_{i}$ the direct product BCC-algebra of $\left\{G_{i}\right\}$. Let $\mu_{i}$ be a $T$-fuzzy multiply positive implicative BCC-ideal of $\left\{G_{i}\right\}$, where $1 \leq i \leq n$. Then $\mu=\prod_{i=1}^{n} \mu_{i}$ defined by $\mu\left(x_{1}, x_{2}, \ldots, x_{n}\right)=\left(\Pi_{i=1}^{n} \mu_{i}\right)\left(x_{1}, x_{2}, \ldots, x_{n}\right)=T_{n}\left(\mu_{1}\left(x_{1}\right), \mu_{2}\left(x_{2}\right), \ldots, \mu_{n}\left(x_{n}\right)\right)$ is a $T$-fuzzy multiply positive implicative BCC-ideal of $G$.

The proof is identical with the corresponding proof from [3].

DEFinition 4.3 [4]. Let $T$ be a $t$-norm and let $\mu$ and $v$ be fuzzy sets in a BCC-algebra $G$. Then the $T$-product of $\mu$ and $\nu$, written as $[\mu \cdot \nu]_{T}$, is defined by $[\mu \cdot v]_{T}(x)=T(\mu(x), v(x))$ for all $x \in G$.

THEOREM 4.4. Let $T$ be at-norm and let $\mu$ and $v$ be $T$-fuzzy multiply positive implicative BCC-ideals of a BCC-algebra $G$. If $T^{*}$ is a $t$-norm which dominates $T$, that is, $T^{*}(T(\alpha, \beta), T(\nu, \delta)) \geq T\left(T^{*}(\nu, \delta), T^{*}(\beta, \delta)\right)$ for all $\alpha, \beta, \nu, \delta \in[0,1]$, then the $T^{*}$-product of $\mu$ and $\nu,[\mu \cdot \nu]_{T^{*}}$, is a $T$-fuzzy multiply positive implicative BCC-ideal of $G$.

Proof. Let $[\mu \cdot v]_{T^{*}}(0)=T^{*}(\mu(0), v(0)) \geq T^{*}(\mu(x), v(x))=[\mu \cdot v]_{T^{*}}(x)$ for any $x \in G$. Moreover, for any $n, m \in \mathbb{N}$, there exists a natural number $k$, such that

$$
\begin{aligned}
{[\mu \cdot v]_{T^{*}}\left(x * z^{k}\right) } \\
\quad=T^{*}\left(\mu\left(x * z^{k}\right), v\left(x * z^{k}\right)\right) \\
\quad \geq T^{*}\left(T\left(\mu\left((x * y) * z^{n}\right), \mu\left(y * z^{m}\right)\right), T\left(v\left((x * y) * z^{n}\right), v\left(y * z^{m}\right)\right)\right) \\
\quad \geq T\left(T^{*}\left(\mu\left((x * y) * z^{n}\right), v\left((x * y) * z^{n}\right)\right), T^{*}\left(\mu\left(y * z^{m}\right), v\left(y * z^{m}\right)\right)\right) \\
\quad=T\left([\mu \cdot v]_{T^{*}}\left((x * y) * z^{n}\right),[\mu \cdot v]_{T^{*}}\left(y * z^{m}\right)\right) .
\end{aligned}
$$

Hence $[\mu \cdot \nu]_{T^{*}}$ is a $T$-fuzzy multiply positive implicative BCC-ideal of $G$.

Let $f: G \rightarrow G^{\prime}$ be an onto homomorphism of BCC-algebras. Let $T$ and $T^{*}$ be $t$-norms such that $T^{*}$ dominates $T$. If $\mu$ and $\nu$ are $T$-fuzzy multiply positive implicative BCC-ideals of $G^{\prime}$, then the $T^{*}$-product of $\mu$ and $\nu,[\mu \cdot \nu]_{T^{*}}$, is a $T$-fuzzy multiply positive implicative BCC-ideal of $G^{\prime}$. Since every onto homomorphism preimage of a $T$-fuzzy multiply positive implicative BCC-ideal is a $T$-fuzzy multiply positive implicative BCC-ideal, the preimages $f^{-1}(\mu), f^{-1}(\nu)$, and $f^{-1}\left([\mu \cdot \nu]_{T^{*}}\right)$ are $T$-fuzzy multiply positive implicative BCC-ideals of $G$. The next theorem provides the relation between $f^{-1}\left([\mu \cdot \nu]_{T^{*}}\right)$ and $T^{*}$-product $\left[f^{-1}(\mu) \cdot f^{-1}(\nu)\right]_{T^{*}}$ of $f^{-1}(\mu)$ and $f^{-1}(\nu)$.

THEOREM 4.5. Let $f: G \rightarrow G^{\prime}$ be an onto homomorphism of BCC-algebras. Let $T$ and $T^{*}$ be $t$-norms such that $T^{*}$ dominates $T$. Let $\mu$ and $v$ be $T$-fuzzy multiply positive implicative BCC-ideals of $G^{\prime}$. If $[\mu \cdot v]_{T^{*}}$ is the $T^{*}$-product of $\mu$ and $\nu$, and $\left[f^{-1}(\mu) \cdot f^{-1}(\nu)\right]_{T^{*}}$ is the $T^{*}$-product of $f^{-1}(\mu)$ and $f^{-1}(\nu)$, then $f^{-1}\left([\mu \cdot v]_{T^{*}}\right)=\left[f^{-1}(\mu) \cdot f^{-1}(\nu)\right]_{T^{*}}$. 
ACKNOWLEDGMENT. The authors would like to thank the referees for their valuable suggestions.

\section{REFERENCES}

[1] M. T. Abu Osman, On some product of fuzzy subgroups, Fuzzy Sets and Systems 24 (1987), no. 1, 79-86.

[2] P. Bhattacharya and N. P. Mukherjee, Fuzzy relations and fuzzy groups, Inform. Sci. 36 (1985), no. 3, 267-282.

[3] W. A. Dudek and Y. B. Jun, On fuzzy BCC-ideals over a t-norm, Math. Commun. 5 (2000), no. 2, 149-155.

[4] W. A. Dudek, K. H. Kim, and Y. B. Jun, Fuzzy BCC-subalgebras of BCC-algebras with respect to a t-norm, Sci. Math. Japon. 3 (2000), no. 1, 99-106.

[5] W. A. Dudek and X. H. Zhang, On ideals and congruences in BCC-algebras, Czechoslovak Math. J. 48(123) (1998), no. 1, 21-29.

[6] Y. Komori, The class of BCC-algebras is not a variety, Math. Japon. 29 (1984), no. 3, 391-394.

[7] X. Ougen, Fuzzy BCK-algebra, Math. Japon. 36 (1991), no. 5, 935-942.

[8] A. Wroński, BCK-algebras do not form a variety, Math. Japon. 28 (1983), no. 2, 211-213.

[9] L. A. Zadeh, Fuzzy sets, Information and Control 8 (1965), 338-353.

Jianming Zhan: Department of Mathematics, Hubei Institute for Nationalities, Enshi, Hubei Province 445000, China

E-mail address: zhanjianming@hotmai 1.com

Zhisong Tan: Department of Mathematics, Hubei Institute for Nationalities, Enshi, Hubei Province 445000, China

E-mail address: es-tzs@263.net 


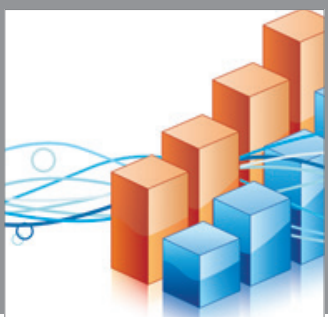

Advances in

Operations Research

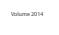

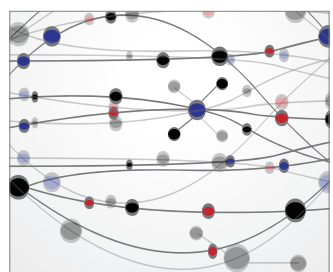

\section{The Scientific} World Journal
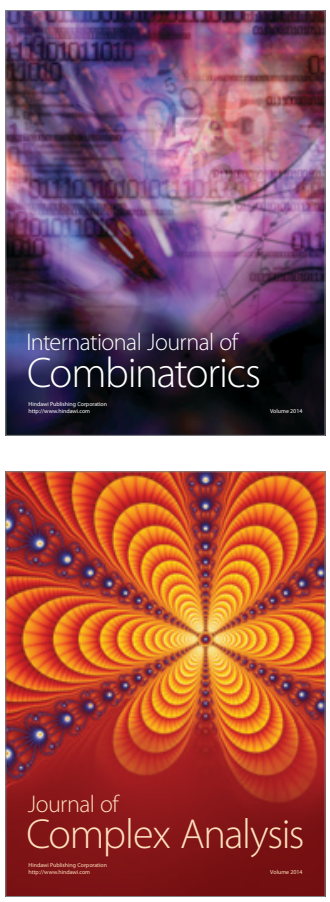

International Journal of

Mathematics and

Mathematical

Sciences
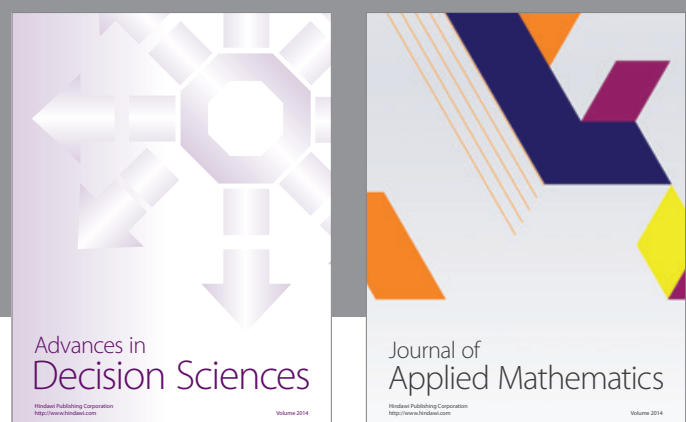

Journal of

Applied Mathematics
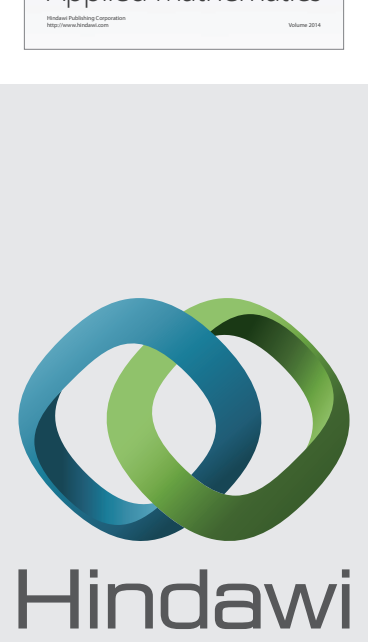

Submit your manuscripts at http://www.hindawi.com
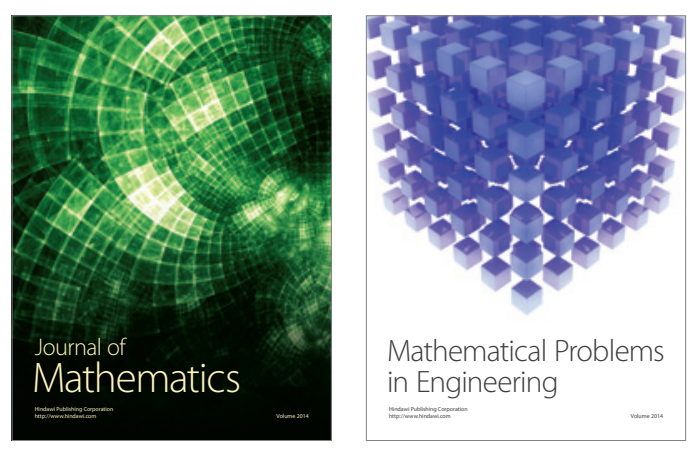

Mathematical Problems in Engineering
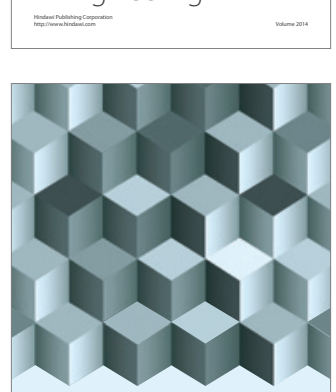

Journal of

Function Spaces
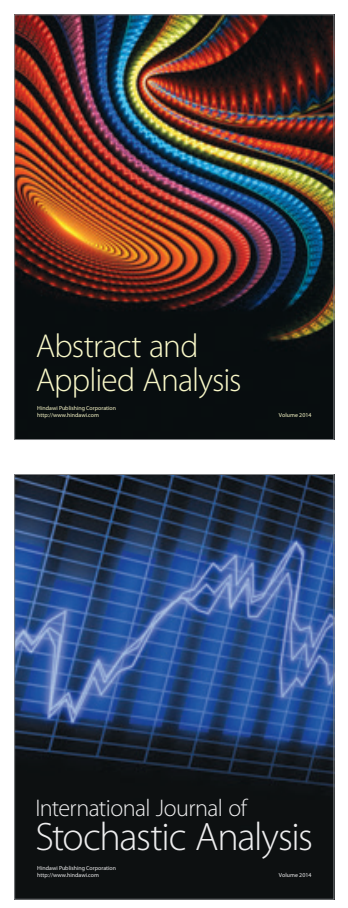

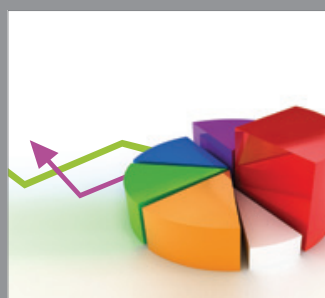

ournal of

Probability and Statistics

Promensencen
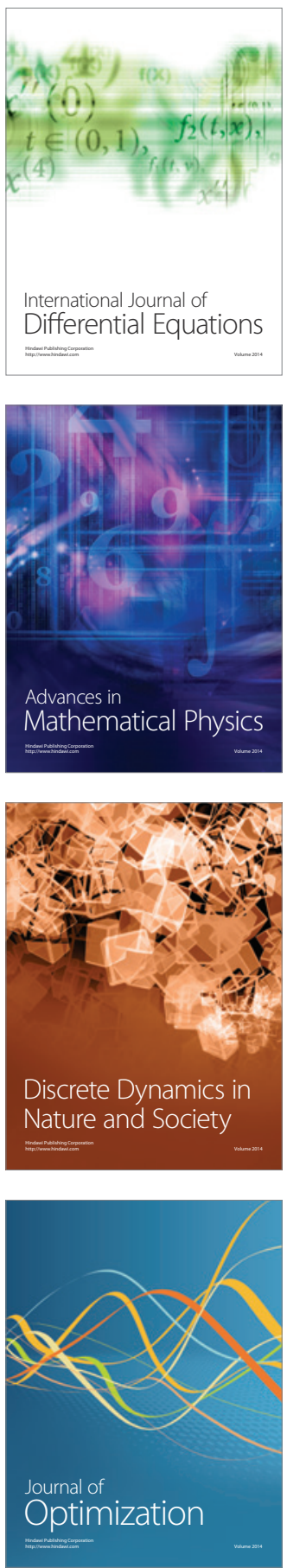